\title{
Nonlinear evolution equations with nonmonotonic perturbations
}

AUTHOR(S):

Hirano, Norimichi

\section{CITATION:}

Hirano, Norimichi. Nonlinear evolution equations with nonmonotonic perturbations. 数理 解析研究所講究録 1988, 647: 57-71

ISSUE DATE:

1988-02

URL:

http://hdl.handle.net/2433/100278

RIGHT: 
Nonlinear evolution equations with

nonmonotonic perturbations

Norimichi Hirano(Yokohama National University) (平㼟给)

1. Introduction. Let $V$ be a Banach space densely and continously imbedded in a real Hilbert space H. Our purpose in this paper is to consider the existence of solutions of the in itial value problem

$$
\frac{d u}{d t}+A u+G(u)=f, \quad 0<t<T,
$$

(1. 1)

$$
u(0)=u_{0}
$$

where $A$ is a monotone operator from $V$ into $V^{\prime}, G: V \rightarrow H$ is a continuous mapping and $f:(0, T) \rightarrow V$, is a measurable function.

Problems of this kind has been studied by many authors. The case $A$ is linear was studied by Browder (5) and Pazy (14). The nonlinear case was studied by Attouch \& Damlamian (1), Crandall \& Nohel (7), Hirano (10), and Vrabie(15, 16). In (15) and (16), Vrabie studied the problem (1. 1) under the assumption that A generates a compact semigroup on $H$, and satisfies

(1.2) (Ax - Ay, $x-y)+c|x-y|^{2} \geq w\|x-y\|^{p}$ for $x, y \varepsilon V$, where $c, \omega>0, p \geq 2$ and $\|\cdot\|,|\cdot|$ denotes the norms of $V$ and $H$, respectively.

In this paper, we consider the case $G$ is a compactly continuous mapping from $V$ into $V^{\prime}$. Our argument is based on the existence results for pseudo-monotone mappings (cf. $(4,6))$. 
2. Statement of main results. Let $p, q$ and $T$ be constants such that $T>0, p \geq 2$ and $1 / p+1 / q=1$. V will denote a reflexive Banach space densely and continuously imbedded in a real Hilbert space H. Identifying $H$ with its dual, we have that $V \subset H \subset V^{\prime}$, where $V^{\prime}$ is the dual space of $V$. the norms of $V, H$ and $V$, are denoted by $\|\cdot\|,|\cdot|$ and $\|\cdot\|_{*}$, respectively. Let $(x, y)$ denote the pairing of an element $x \varepsilon V$ and an element $y \varepsilon V^{\prime}$. If $x, y \varepsilon H$, then $(x, y)$ is the ordinary inner product of $H$. Let $A$ be a mapping from $V$ into $V^{\prime}$. Then $A$ is called monotone if $(A x-A y, x-y) \geq 0$ for $x, y \varepsilon V$. the mapping $A$ is said to be hemicontinuous if for each $x, y \varepsilon V, A(u+t v)$ converges to $A u$ weakly in $V$, as $t \rightarrow 0$. A is called pseudo-monotone if $A$ satisfies the following condition:

(2.2) If $\left\{u_{n}\right\}$ is a sequence such that $u_{n}$ converges weakly to $u \varepsilon V$ and $l i m \sup \left(A u_{n}, u_{n}-u\right) \leq 0$, then

$$
(A u, u-v) \leq \underset{n \rightarrow \infty}{\lim } \inf _{n \rightarrow \infty}\left(A_{n}, u_{n}-v\right) \text { for each } v \varepsilon V \text {. }
$$

Let $E, F$ be Banach spaces, and let $g$ be a mapping from $E$ into $F$. We denote by $E_{w}$ and $F_{w}$ the spaces $E$ and $F$ endowed with their weak topologies, respectively. Then $g$ is said to be weakly continuous if $g$ is a continuous mapping from $E_{w}$ into $F_{w}$. The mapping $g$ is called demicontinuous if $g$ is a continuous mapping from $E$ into $F_{w}$. For each $r \geq 1$. We denote by $L^{r}(0, T ; E)$ the space of E-valued measurable functions $u:(0, T) \rightarrow E$ such that $\int_{0}^{T}\|u(t)\|^{r} d t<\infty$. The pairing between $L^{p}(0, T ; V)$ and $L^{q}\left(0, T ; V^{\prime}\right)$ is denoted by $<\cdot, \cdot>$. Then for each $u, v \in L^{2}(0, T ; H),\langle u, v>$ is the ordinary innner product 
of $u$ and $v$ in $L^{2}(0, T ; H)$. The norms of $L^{p}(0, T ; V), L^{2}(0, T ; H)$, $L^{q}\left(0, T ; V^{\prime}\right)$ are again denoted by $\|\cdot\|,|\cdot|$ and $\|\cdot\|_{*}$. We denote by $J$ the duality mapping from $L^{q}\left(0, T ; V^{\prime}\right)$ on to $L^{p}(0, T ; V)$, i.e.,

(2. 1) $\mathrm{J}(\mathrm{u})=\left\{\begin{array}{lll}\mathrm{v} & \varepsilon \mathrm{L}^{\mathrm{p}}(0, \mathrm{~T} ; \mathrm{V}):\langle\mathrm{v}, \mathrm{u}\rangle=\|\mathrm{v}\|^{2}=\|\mathrm{u}\|_{*}^{2}\end{array}\right\}$

for each $u \in L^{q}\left(0, T: V^{\prime}\right)$. By using the Asplund's renorming theorem, we may assume that $\mathrm{J}$ is a single valued monotone and demicontinuous mapping(cf. Proposition 2.14 of (3)). We will denote by $L$ the operator defined by

$$
(L f)(t)=\int_{0}^{t} f(s) d s \quad \text { for each } f \in L^{2}((0, T))
$$

The adjoint operator $L^{*}$ of $L$ is given by

$$
\left(L^{*} f\right)(t)=\int_{t}^{T} f(s) d s \quad \text { for each } f \in L^{2}((0, T)) \text {. }
$$

Then $L$ and $L^{*}$ are positive operators on $L^{2}((0, T))$.

In the following we will assume that the mapping $A: V \rightarrow V^{\prime}$ satisfies the following conditions:

(A1) A is a monotone hemicontinuous mapping from $V$ into $V^{\prime}$;

(A2) there exist positive constants $C_{1}, C_{2}$ and $C_{3}$ such that

(2. 3) $\|A x\|_{*} \leq C_{1}\left(\|x\|^{p-1}+1\right)$, for each $x \in V$

and

(2. 4)

$$
C_{2}\|x\|^{p} \leq C_{3}+(A x, x) \quad \text { for each } x \in V
$$

We impose the following conditions on G:

(G1) $G$ is a completely continuous mapping from $V$ to $V$ :

(G2) There exist positive constants $a, b$ and $C$ such that 
(2. 5) $\quad(G(x), x) \geq-C$

(2.6) $\quad\|G(x)\|_{*} \leq a\|x\|^{p-1}+b$ for a $11 \times \varepsilon V$;

for a $11 \times \varepsilon V$.

We now state our result:

Theorem. Suppose that (A1), (A2), (G1) and (G2) hold. Then for each $u_{0} \varepsilon H$ and $f \in L^{q}\left(0, T ; V^{\prime}\right)$, there exists a solution $u$ of (1. 1) such that

$$
u \varepsilon C(0, T ; H) \cap L^{p}(0, T ; V)
$$

(2. 8) $\quad \frac{d u}{d t} \varepsilon L^{q}\left(0, T: V^{\prime}\right)$.

3. Propositions. Throughout this section, we assume that

$u_{0} \varepsilon V, f \in L^{q}\left(0, T ; V^{\prime}\right)$, and that (A1), (A2), (G1) and (G2) hold We denote by $\widetilde{V}, \widetilde{H}$, and $\widetilde{V}$, the spaces $L^{p}(0, T ; V), L^{2}(0, T ; H)$ and $L^{q}\left(0, T ; V^{\prime}\right)$, respectively. $\tilde{A}$ denote the operator defined by

$$
(\tilde{A} u)(t)=A\left(u(t)+u_{0}\right)-f(t), \text { for each } u \varepsilon \tilde{V} \text { and } t \varepsilon(0, T)
$$

Also we denote by $\widetilde{G}$ the mapping defined by

$$
(\widetilde{G} u)(t)=G\left(u(t)+u_{0}\right) \quad \text { for each } u \in \tilde{V} \text { and } t \varepsilon(0, T) \text {. }
$$

Then it is easy to see that $\widetilde{A}$ is a monotone hemicontinuous mapping satisfying the following conditions:

(3. 1) $\quad\|\tilde{A} u\|_{*} \leq c_{1}\left(1+\|u\|^{p-1}\right) \quad$ for $u \varepsilon \tilde{V}$;

(3. 2) $\quad c_{2}\|u\|^{p} \leq c_{3}+<\tilde{A} u, u>\quad$ for $u \varepsilon \tilde{V}$, 
where $c_{1}, \quad c_{2}$ and $c_{3}$ are positive constants depending on $\mathrm{C}_{1}, \mathrm{C}_{2}$, $\mathrm{C}_{3}, \mathrm{~T}, \mathrm{u}_{0}$ and $\mathrm{f}$. It is also easy to see that $\mathrm{G}$ is a continuous mapping from $\widetilde{V}$ into $\widetilde{H}$ satisfying that

(3. 3) $<\tilde{G u}, u>\geq c \quad$ for all $u \in \tilde{V}$;

(3. 4) $\|\widetilde{G} u\|_{\dot{x}} \leq \alpha\|u\|^{p-1}+B \quad$ for all u $\varepsilon \tilde{V}$,

where $c, \alpha, B$ are constants depending on $C, a, b$ and $T$ and $u_{0}$. We now consider the equation of the form

(3. 5) $\quad v+(\tilde{A}+\tilde{G}) L v=0$

Let $v \varepsilon \widetilde{V}$, be a solution of (3. 5). Then it is easy to see that $u=L v+u_{0}$ is a solution of (1.1). On the other hand, if $u$ is a solution of (1.1), we have that $v=\frac{d u}{d t}$ is a solution of (3.4). Since $L^{*}$ is injective, the equation (3.5) is equivalent to (3. 6) $\quad L^{*} v+L^{*}(\tilde{A}+\widetilde{G}) L v=0$.

Then we will show the existence of the solutions of (3.6) instead of (1.1). In the rest of this section, we assume, for simplicity, that $u_{0}=0$ and $f=0$. The proofs remains valid for each $u_{0} \varepsilon V$ and $f \in \widetilde{V}^{\prime}$ with minor changes.

Proposition 1. the mapping $L^{*}+L^{*}(\tilde{A}+\widetilde{G}) L$ is a pseudomonotone mapping from $\tilde{V}$ into $\widetilde{V}$ '. 
Proof. From (A1), it is easily verified that $L^{*}+L^{*} \tilde{A} L: \widetilde{V} \rightarrow \tilde{V}$. is a monotone hemicontinuous mapping. Let $\left\{v_{n}\right\} \subset \tilde{V}$ be a sequence such that $v_{n}$ converges to $v$ weakly in $\widetilde{V}$ and

$$
\lim \sup <L^{*} v_{n}+L^{*}(\tilde{A}+\widetilde{G}) L v_{n}, v_{n}-v>\leq 0 .
$$

Since $v_{n}$ converges to $v$ weakly in $\tilde{V}$, we have that for each $t \quad \varepsilon \quad(0, T),\left(L_{n}\right)(t)$ converges to $(L v)(t)$ weakly in $V$. Since $G$ is completely continuous, we find that $G\left(\left(\operatorname{Lv}_{n}\right)(t)\right)$ converges to $G((L v)(t))$ strongly in $V^{\prime}$ for all $t \in(0, T)$. Then noting that

$$
\left\|G\left(\left(L v_{n}\right)(t)\right)\right\|_{*} \leq a\left\|\left(L v_{n}\right)(t)\right\|^{p-1}+b \leq a\left(T^{1 / 2} s u p\left\|v_{n}\right\|\right)^{p-1}+b
$$

for each $t \varepsilon(0, T)$, we obtain by Lebesgue's bounded convergence theorem, that $\widetilde{G}\left(L_{n}\right)$ converges to $\widetilde{G}(L v)$ strongly in $\widetilde{v}$. Thus we obtain that

(3. 8) $<\widetilde{G} L v, L v>=1 i m<\widetilde{G} L v_{n}, L_{n}>$

Therefore we have by (3.7) and (3.8) that

$$
\lim \sup \left\langle L^{*} v_{n}+L^{*} \tilde{A} L v_{n}, v_{n}-v>\leq 0\right.
$$

Then by lemma 1.3 of Chap II of (2), it follows that $L^{*} v_{n}+L^{*} \tilde{A} L v_{n}$ converges to $L^{*} v+L^{*} \tilde{A} L v$ weakly in $\tilde{V}$, and

$$
\left\langle L^{*} v+L^{*} \tilde{A} L v, v>=1 i m\left\langle L^{*} v_{n}+L^{*} \tilde{A} L v_{n}, v_{n}\right\rangle .\right.
$$

Then from (3.7), (3. 8) and the equality above, we find that

$$
\left\langle L^{*} v+L^{*}(\tilde{A}+\tilde{G}) L v, L v-z>\leq l i m \text { inf }\left\langle L^{*} v_{n}+L^{*}(\tilde{A}+\widetilde{G}) L v_{n}, v_{n}-z\right\rangle\right.
$$

for each $z \in \tilde{V}$. This completes the proof. 
Proposition 2. Let $\left\{v_{n}\right\}$ be a sequence in $\tilde{V}$, such that $v_{n}$ converges to $v$ weakly in $\tilde{V}^{\prime},\left\{L v_{n}\right\} \subset \tilde{V}, L v_{n}$ converges to Lv weakly in $\widetilde{V}$ and

$$
\lim \sup \left\langle(\widetilde{A}+\widetilde{G}) L v_{n}, L v_{n}-L v>\leq 0\right.
$$

Then $(\tilde{A}+\widetilde{G}) L v_{n}$ converges to $(\tilde{A}+\widetilde{G}) L v$ weakly in $\tilde{V}$, and (3. 9) $\quad \lim \left\langle(\tilde{\mathrm{A}}+\widetilde{G}) L v_{n}, \operatorname{Lv}_{n}\right\rangle=\langle(\tilde{A}+\widetilde{G}) L v, L v\rangle$

Proof. Let $\left\{v_{n}\right\}$ be a sequence in $\widetilde{V}^{\prime}$ satisfying the hypothesis of Proposition 2. Then by using (3.1) and (3.4), we can see that that $\left\{\left\|\tilde{A} L v_{n}\right\|_{*}\right\}$ and $\left\{\left\|\widetilde{G} L v_{n}\right\|_{*}\right.$ are bounded. We first show that (3. 10) $\lim$ inf $\left(A\left(\left(L v_{n}\right)(t)\right)+G\left(\left(L v_{n}\right)(t)\right),\left(L v_{n}-L v\right)(t)\right) \geq 0$ for all t $\varepsilon(0, T)$. Suppose that for some $t \varepsilon(0, T)$, (3. 11) $\quad$ im inf $\left(A\left(\left(\operatorname{Lv}_{n}\right)(t)\right)+G\left(\left(\operatorname{Lv}_{n}\right)(t)\right),\left(\operatorname{Lv}_{n}-\operatorname{Lv}\right)(t)\right)<0$. From (A2) and (G2), we have that

(3. 12)

$$
\begin{aligned}
\left(A\left(\left(L v_{n}\right)(t)\right)+G\left(\left(L v_{n}\right)(t)\right),\right. & \left.\left(L v_{n}-L v\right)(t)\right) \\
\geq C_{2}\left\|\left(L v_{n}\right)(t)\right\|^{p}-C_{3} & -C-C_{1}\left(1+\left\|\left(L v_{n}\right)(t)\right\|^{p-1}\right)\|(L v)(t)\| \\
& -\left(a\left\|\left(L v_{n}\right)(t)\right\|^{p-1}+b\right)\|(L v)(t)\| .
\end{aligned}
$$

Then it follows from (3.11) and (3.12) that $\left\{\left\|\left(L_{n}\right)(t)\right\|\right\}$ is bounded. Then since $G$ is compltetly continuous, $G\left(\mathrm{Lv}_{n}\right)(t)$ converges to $G(L v)(t)$ strongly in $V^{\prime}$. Therefore we have that $\lim \left(G\left(\left(\operatorname{Lv}_{n}\right)(t)\right), \quad\left(\operatorname{Lv}_{n}\right)(t)-(L v)(t)\right)=0$. On the other hand, we have from the monotonicity of A that

$$
\lim \text { inf }\left(A\left(\left(L v_{n}\right)(t)\right),\left(L v_{n}-L v\right)(t)\right) \geq 0
$$


for all t $\varepsilon(0, T)$. Then we have that

$\lim$ inf $\left(A\left(\left(L v_{n}\right)(t)\right)+G\left(\left(L v_{n}\right)(t)\right), \quad\left(L v_{n}-L v\right)(t)\right) \geq 0$

This contradicts to (3.11). Thus we have shown that (3.10) holds for all t $\varepsilon(0, T)$. We can see from (3. 12) that

$$
\begin{aligned}
h_{n}(t)=\left(A\left(\left(L v_{n}\right)(t)\right)+G\left(\left(L v_{n}\right)(t)\right),\left(L v_{n}\right.\right. & -L v)(t)) \\
& \geq K_{1}\|L v(t)\|^{p}+K_{2}
\end{aligned}
$$

for all $t \in(0, T)$ and $n \geq 1$, where $K_{1}, K_{2}$ are constants depending on $C, C_{1}, C_{2}, C_{3}, a$, and $b$. Then by Fatou's lemma, we have that

(3. 13) $0=\int_{0}^{T} 1$ im inf $h_{n}(t) d t$

$$
\leq \lim \text { inf } \int_{0}^{T} h_{n}(t) d t \leq l \text { im sup }<(\tilde{A}+\tilde{G}) L v_{n}, L v_{n}-L v>\leq 0
$$

The inequality above implies that $l \mathrm{im} \int_{0}^{\mathrm{T}}\left|\mathrm{h}_{n}\right| \mathrm{d} t=0$. Then we can choose a subsequence $\left\{h_{n_{i}}\right\}$ of $\left\{h_{n}\right\}$ such that

(3. 14) $\lim \left(A\left(\left(\operatorname{Lv}_{n_{i}}\right)(t)\right)+G\left(\left(\operatorname{Lv}_{n_{i}}\right)(t)\right),\left(\operatorname{Lv}_{n_{i}}-L v\right)(t)\right)=0$,

a.e. $t \varepsilon(0, T)$. By (3.12) and (3.14), we find that $\left\{\left\|L v_{n_{i}}(t)\right\|\right\}$ is bounded for a.e. $t \in(0, T)$. Since $\left(L_{n_{i}}\right)(t)$ converges to $(L v)(t)$ weakly in $V^{\prime}$, we have that $G\left(L v_{n_{i}}\right)(t)$ converges to $G(L v)(t)$ strongly in $H$, for a.e. $t \varepsilon(0, T)$. Therefore it follows from (2.6) that

$\lim \left(G\left(\left(L v_{n_{i}}\right)(t)\right),\left(\operatorname{Lv}_{n_{i}}\right)(t)-(L v)(t)\right)=0$. Then we have

$$
\lim \left(A\left(\left(L v_{n_{i}}\right)(t)\right),\left(L_{n_{i}}-L v\right)(t)\right)=0 \text { a.e. } t \varepsilon(0, T)
$$


Then since $A$ is monotone, we have from lemma 1.3 of Chap. II of (2) that $A\left(L_{n_{i}}(t)\right)$ converges to $\left.A(C L)(t)\right)$ weakly in $V^{\prime}$. Here we observe by using (3. 1)-(3.4) that for each z $\varepsilon \tilde{V}$ and $t \varepsilon(0, T)$, there exist real numbers $K_{3}, K_{4}$ such that

(3.15) $\left((A+G)\left(\left(\operatorname{Lv}_{n}\right)(t)\right),\left(\operatorname{Lv}_{n}\right)(t)-z(t)\right) \geq K_{3}\|z(t)\|^{p}+K_{4}$ for each $n \geq 1$. Then from Fatou's lemma, we find that

(3. 16) $<(\tilde{A}+\widetilde{G}) L v, L v-z>$

$$
\begin{aligned}
& =\int_{0}^{T} \lim \left(A\left(\left(\operatorname{Lv}_{n_{i}}\right)(t)\right)+G\left(\left(\operatorname{Lv}_{n_{i}}\right)(t)\right),\left(\operatorname{Lv}_{n_{i}}\right)(t)-z(t)\right) d t \\
& \leq \lim \inf \left\langle(\tilde{A}+\tilde{G}) L_{n_{i}}, L_{n_{i}}-z\right\rangle \\
& \leq \lim \sup \left\langle(\tilde{A}+\widetilde{G}) L v_{n},\left(L v_{n}-L v\right)+(L v-z)\right\rangle \\
& \leq \lim \sup \left\langle(\tilde{A}+\tilde{G}) L v_{n}, L v-z>, \text { for all z } \varepsilon \tilde{V}\right. \text {. }
\end{aligned}
$$

The inequality above implies that $(\tilde{A}+\widetilde{G}) L v_{n}$ converges to $(\widetilde{A}+\widetilde{G}) L v$ weakly in $\tilde{V}^{\prime}$. We also obtain from (3. 13) that (3.9) holds.

Proposition 3. For each $k>0$, the equation

(3. 17)

$$
L^{*} v+k J v+L^{*}(\tilde{A}+\tilde{G}) L v=0
$$

has a solution $v \in \widetilde{V}^{\prime}$.

Proof. Let $B_{r}=\{v \varepsilon \tilde{V}:\|v\| \leq r\}$ for $r>0$. Since the mapping $L^{*}+L^{*}(\tilde{A}+\tilde{G}) L: \tilde{V} \rightarrow \tilde{V}$, is pseudo-monotone and $J: \tilde{V} \rightarrow \tilde{V}\left(C \tilde{V} \tilde{V}^{\prime}\right)$ is 
monotone hemicontinuous, we can see that the sum $k J+L^{*}+L^{*}(\tilde{A}+\widetilde{G}) L$ is also pseudo-monotone (cf. Proposition 23 of (4)), for each

$k>0$. Then we have, by using theorem 7.8 of (6), that for each $n$ $z 1$, there exists a solution $v_{n} \varepsilon B_{n}$ of the inequality

(3. 18) $<L^{*} v_{n}+k J v_{n}+L^{*}(\tilde{A}+\widetilde{G}) L v_{n}, z-v_{n}>\geq 0$ for all $z \varepsilon B_{n}$.

The inequlity (3. 18) implies that for each $m \geq 1$,

(3. 19) $\quad \lim _{n \rightarrow \infty} \sup \left(<v_{n}+(\tilde{A}+\tilde{G}) L v_{n}, L v_{n}-L v_{m}>+k<J v_{n}, v_{n}-v_{m}>\right)$ $=1 \mathrm{im} \sup _{n \rightarrow \infty}<L^{*} v_{n}+k J v_{n}+L^{*}(\widetilde{A}+\widetilde{G}) L v_{n}, v_{n}-v_{m}>\leq 0$.

By putting $v=0$ in (3. 18), we have

(3. 20) $\quad k\left\|v_{n}\right\|_{*}^{2}+c_{2}\left\|L v_{n}\right\|^{p}+c \leq c_{3}$ for all $n \geq 1$

The inequality above implies that $\left\{\left\|v_{n}\right\|_{*}\right\}$ and $\left\{\left\|L v_{n}\right\|\right\}$ are bounded. Then we may assume without any loss of generality that $v_{n}$ converges to $v \in \tilde{V}$, weakly in $\tilde{V}^{\prime}$ and $L v_{n}$ converges to Lv weakly in $\tilde{V}$. Then from (3. 19), it is easy to see that

(3. 21) $\lim \sup \left(<v_{n}+(\tilde{A}+\widetilde{G}) L v_{n}, L v_{n}-L v>+k\left\langle J v_{n}, v_{n}-v>\right) \leq 0\right.$.

Here we choose a sequence $\left\{z_{n}\right\} \subset \tilde{V}$, such that $z_{n} \varepsilon \operatorname{co}\left\{v_{n}\right\}, z_{n}$ converges to $v$ strongly in $\tilde{V}$, and $L z_{n}$ converges to Lv strongly in V. Then since $<L v_{n}-L z_{m}, v_{n}-z_{m}>\geq 0$, we find, by letting $\mathrm{m}, \mathrm{n} \rightarrow \infty$ that

(3. 22) $\quad l i m$ inf $<v_{n}, L v_{n}-L v>\geq 0$.

Also we have by the monotonicity of $J$ that 
(3. 23) $\quad \mathrm{im}$ inf $\left\langle\mathrm{J} \mathrm{v}_{\mathrm{n}}, \mathrm{v}_{\mathrm{n}}-\mathrm{v}\right\rangle \geq 0$.

Combining (3.22) and (3.23) with (3.21), we have

(3. 23) $\lim \sup <(\tilde{A}+\widetilde{G}) L v_{n}, L v_{n}-L v>\leq 0$.

Then we obtain by Proposition 2 that $(\tilde{A}+\widetilde{G}) L v_{n}$ converges to $(\tilde{A}+\widetilde{G}) L v$ weakly in $\widetilde{V}$, and

(3. 24) $\left.1 \mathrm{im}<(\tilde{A}+\widetilde{G}) L v_{n}, L v_{n}\right\rangle=\langle(\tilde{A}+\tilde{G}) L v, L v\rangle$.

Then the inequality (3. 18) implies that

(3. 25) $\left\langle L^{*} v+k J v+L^{*}(\tilde{A}+\widetilde{G}) L v, z\right\rangle z\langle(\tilde{A}+\widetilde{G}) L v, L v>$ for all z $\varepsilon \tilde{v}$. Since $z \varepsilon \tilde{V}$ is arbitrary, we find that $L^{*} v+k J v+L^{*}(\tilde{A}+\widetilde{G}) L v=0$.

4. Proof of Theorems. In the following, we assume that (A1), (A2), (G1) and (G2) are satisfied. We first show that the assertion of Theorem 1 holds for each $u_{0} \varepsilon V$ and $f \varepsilon \tilde{V}^{\prime}$.

Let $u_{0} \quad \varepsilon \quad V, \quad f \quad \varepsilon \quad \tilde{V}$ and let $\tilde{A}, \tilde{G}$ be as in section 3 . Then by Proposition 3 , there exists a solution $v_{n} \varepsilon \tilde{V}^{\prime}$ of the equation

(4. 1)

$\mathrm{L}^{*} \mathrm{v}_{\mathrm{n}}+\frac{1}{\mathrm{n}} \mathrm{J} \mathrm{v}_{\mathrm{n}}+\mathrm{L}^{*}(\tilde{\mathrm{A}}+\widetilde{\mathrm{G}}) \mathrm{L} \mathrm{v}_{\mathrm{n}}=0$

for each $n \geq 1$. Multiplying (4.1) by $v_{n}$, we find that

(4. 2) $\quad \frac{1}{n}\left\|v_{n}\right\|_{*}^{2}+c_{2}\left\|L v_{n}\right\|^{p}+c \leq c_{3} \quad$ for each $z \geq 1$.

From (4.2), we have that $\left\{\left\|L v_{n}\right\|\right\}$ is bounded. Then it follows from

(3. 1) and (3.4) that $\left\{\left\|\tilde{A} L v_{n}\right\|_{*}\right\}$ and $\left\{\left\|\tilde{G} L v_{n}\right\|_{*}\right\}$ are bounded. It also 
follows from (4.2) that $1 \mathrm{im}\left\|\frac{1}{n} \mathrm{~J} v_{n}\right\|=0$. Since $L^{*}$ is injective in $\tilde{V}^{\prime}$, the equation (4. 1) can be rewritten as

(4. 3) $\quad v_{n}+\frac{1}{n}\left(L^{*}\right)^{-1} J v_{n}+(\tilde{A}+\widetilde{G}) L v_{n}=0 \quad$ for each $n \geq 1$.

Here we note that $\left\langle\left(L^{*}\right)^{-1} \mathrm{Jv}_{\mathrm{n}}, J \mathrm{Jv}_{\mathrm{n}}\right\rangle \geq 0$ for $\mathrm{n} \geq 1$. Then multiplying (4.3) by $\mathrm{Jv}_{\mathrm{n}}$, we have

(4.4) $\quad\left\|v_{n}\right\|_{*}^{2} \leq\left\|(\tilde{A}+\widetilde{G}) L v_{n}\right\|_{*}\left\|J v_{n}\right\| \leq\left(\left\|\widetilde{A} L v_{n}\right\|_{*}+\left\|\widetilde{G} L v_{n}\right\|\right)_{*}\left\|v_{n}\right\|_{*}$.

for $n \geq 1$. Thus we find that $\left\{\left\|v_{n}\right\|_{*}\right\}$ is bounded. Then we may suppose without any loss of generality that $v_{n}$ converges to $v \varepsilon \tilde{V}$, weakly in $\tilde{V}^{\prime}$ and $L v_{n}$ converges to Lv weakly in $\tilde{V}$.

While, we have by multiplying (4.1) by $v_{n}-v_{m}$ that

(4.5) $<v_{n}+(\tilde{A}+\tilde{G}) L v_{n}, L v_{n}-L v_{m}>+<\frac{1}{n} J v_{n}, v_{n}-v_{m}>=0$.

Then since $\frac{1}{n} J v_{n}$ converges to 0 in $\tilde{V}$ as $n \rightarrow \infty$, we find that

(4.6) $\quad \lim _{n \rightarrow \infty}<v_{n}+(\tilde{A}+\widetilde{G}) L v_{n}, L v_{n}-L v_{m}>=0$ for each $m \geq 1$.

Then it is easy to see from (4.6) that

(4.7) $\quad \lim \left\langle v_{n}+(\tilde{A}+\widetilde{G}) L v_{n}, L v_{n}-L v>=0\right.$

Since $l i m$ inf $\left(v_{n}, L v_{n}-L v>\geq 0\right.$, we have by (4.7) that

(4. 8) $\quad \lim \sup <(\tilde{A}+\widetilde{G}) L v_{n}, L v_{n}-L v>\leq 0$.

Then by Propostion 2 , we find that $(\tilde{A}+\widetilde{G}) L v_{n}$ converges to $(\tilde{A}+\widetilde{G}) L v$ weakly in $\tilde{V}^{\prime}$. Therefore we obtain from (4.1) that

$L^{*} v+L^{*}(\tilde{A}+\tilde{G}) L v=0$, i. e., (1.1) has a solution. 
Now let $u_{0} \varepsilon H$ and $\left\{u_{n}^{0}\right\} \subset V$ be a sequece such that $u_{n}^{0}$ converges to $u_{0}$ strongly in $H$. Then by the argument above, we have that for each $n \geq 1$, there exists a solution $u_{n}$ of the problem

(4. 9)

$$
\frac{d u_{n}}{d t}+A u_{n}+G\left(u_{n}\right)=f, \quad 0<t<T \text {, }
$$

$$
u(0)=u_{n}^{0} \text {. }
$$

Here we assume for simplicity that $f=0$. Then multiplying (4.9) by $u_{n}$ and integrating, we have

(4. 10) $\quad \frac{1}{2}\left|u_{n}(t)\right|^{2}+C_{2} \int_{0}^{T}\left\|u_{n}(s)\right\|^{p} d s<\left(C+C_{3}\right) T+\sup _{n}\left|u_{n}^{0}\right|^{2}$.

Then $\left\{u_{n}\right\}$ is bounded in $\tilde{v}$. Also by (2.3) and (2.6), we see that $\left\{\frac{d u_{n}}{d t}\right\}$ is bounded in $\tilde{V}^{\prime}$. Here we put $v_{n}=\frac{d u_{n}}{d t}$ for each $z \geq 1$. Then from the observation above, we may suppose that $v_{n}$ converges to $v \varepsilon \tilde{V}^{\prime}$ weakly in $\tilde{V}$, and $u_{n}=L v_{n}+u_{n}^{0}$ converges to $u=L v+u_{0}$ weakly in $\tilde{V}$. We set $(\bar{A} z)(t)=A(z(t))$ and $(\bar{G} z)(t)=G(z(t))$ for each $z \quad \varepsilon \quad \tilde{V}$ and $t \varepsilon(0, T)$. Now we multiply (4.9) by $u_{n}-u$ and integrate. Then we have

(4. 11) $\left.\lim \sup <(\bar{A}+\bar{G})\left(\mathrm{Lv}_{n}+u_{n}^{0}\right),\left(\mathrm{Lv}_{\mathrm{n}}+\mathrm{u}_{\mathrm{n}}^{0}\right)-\left(\mathrm{Lv}+\mathrm{u}_{0}\right)\right\rangle$

$$
\begin{aligned}
& =\lim \sup <\frac{d u_{n}}{d t}, u-u_{n}> \\
& \leq \lim \sup \left(-\left|u(T)-u_{n}(T)\right|^{2}+\left|u_{0}-u_{n}^{0}\right|^{2}+<\frac{d u}{d t}, u_{n}-u>\right) \leq 0 .
\end{aligned}
$$

Therefore the hypothesis of Proposition 2 is satisfied with Lv replaced by $L v_{n}+u_{n}^{0}$ and $L v$ replaced by $L v+u_{0}$. It is easy to verify that the proof of Propostition 2 remains valid for A, G, Lv 
and $L_{n}$ replaced by $\bar{A}, \quad \bar{G}, \quad L v+u_{0}$ and $L v_{n}+u_{n}^{0}$, respectively. Therefore we find that $(\bar{A}+\bar{G})\left(L_{n}+u_{n}^{0}\right)$ converges to $(\bar{A}+\bar{G})\left(L v+u_{0}\right)$ weakly in $\tilde{V}$. Thus we obtain $v+(\bar{A}+\bar{G})\left(L v+u_{0}\right)=0$. This implies that $u=L v+u_{0}$ is a solution of (1.1). We can see that $u \varepsilon C(0, T ; H)$ by the usual argument (cf. theorem 4.5 of (3)),

\section{REFERENCES}

(1) Attouch A. \& Damlamian A., On multivalued evolution equations in Hilbert spaces, Is rael J. Math., 12(1972), 373-390.

(2) Barbu V., Nonlinear semigroups and evolution equations in Banach spaces, Noordhoff, Leyden (1976).

(3) Barbu V., \& Precupanu Th., Convexity and Optimization in Banach Spaces, Editura Academiei R. S. R., Bucharest (1975).

(4) Brezis H., Equations et inequations non lineaires dans les espaces vectoriels en dualite, Ann. Inst. Fourier. Grenoble $18(1968), \quad 115-175$.

(5) Browder F. E., Non-linear equations of evolution, Ann. Math., $80(1964), 485-523$.

(6) Browder F. E., Nonlinear Operators and Nonlinear Equations of Evolution in Banach spaces, Proceedings of Symposia in Pure Math. , XVIII, Part 2(1976).

(7) Crandall M. G. \& Nohel J. A., An abstract functional differential equation and a related Volterra equation, Is rael 
J. Math., $29(1979), 313-328$.

(8) Gutman, S., Compact perturbations of m-accretive operators in general Banach spaces, SIAM J. Math., 13(1982), 789-800.

(9) Gutman, S., Evolutions governed by m-accretive plus compact operators, Nonlinear Analys is TMA, 7(1983), 707-715.

(10) Hirano, N., Local existence theorems for nonlinear differential equations, SIAM J. Math. Anal., 14 (1983), 117125.

(11) Hirano, N., Abstract nonlinear Volterra equations with positive kernels, SIAM J. Math. Anal., 17 (1986), 403-414.

(12) Pavel N. H., Invariant sets for a class of semi linear equations of evolutions, Nonlinear Analysis TMA, 1 (1977), 187-196.

(13) Pavel N. H., Equations d'evolution multivoques dans des espaces de Banach, C. r. hebd. Seanc. Acad. Sci. Paris $287(1978), 315-317$.

(14) Pazy, A., A class of semi-linear equations of evolution, Is rael J. Math., 20 (1975), 23-36.

(15) Vrabie, I. I., The nonlinear version of Pazy's local existence theorem, Israel J. Math., 32(1979), 221-235.

(16) Vrabie, I. I., An existence result for a class of nonlinear evolution equations in Banach spaces, Nonlinear Analys is, $6(1982), 711-722$.

(17) Vrabie, I. I, Compactness methods for an abstract nonlinear Volterra integrodifferential equations, Nonlinear Analys is TMA, $5(1981), 355-371$. 